\title{
WiLliaM STANLEY JEVONS, UN PIONNIER DES RÉFLEXIONS SUR LA FISCALITÉ ÉCOLOGIQUE
}

\author{
Antoine MISSEMER ${ }^{*}$
}

Reference (to cite the paper):

Missemer, Antoine. 2013. «William Stanley Jevons, un pionnier des réflexions sur la fiscalité écologique ». L'Économie politique, vol. 60, p. 78-90.

[http://dx.doi.org/10.3917/leco.060.0078]

The pages of the published version are indicated in the margins.

\section{Abstract}

\footnotetext{
* [In 2013] Centre Walras-Pareto, University of Lausanne, Internef, CH-1015 Lausanne-Dorigny \& Triangle, University of Lyon 2, ISH, 14 av. Berthelot, F-69007 Lyon. E-mail address: antoine.missemer@unil.ch
} 
En plaçant la transition énergétique au cœur des futurs « investissements d'avenir » et en établissant une nouvelle «contribution carbone», le gouvernement français a remis à l'agenda politique la question environnementale. Parmi les mesures répondant à cet agenda, la fiscalité écologique occupe une place sensible, en particulier depuis la censure par le Conseil constitutionnel d'un premier dis- positif visant l'instauration d'une taxe carbone, fin 2009. De son élaboration à son abandon au printemps 2010, la taxe carbone a suscité dans l'Hexagone des débats passionnés, parfois empreints de technicité, mais dépassant souvent le seul cadre de la fiscalité écologique pour en venir à des questions de justice sociale et de compétitivité internationale.

Bien que l'enjeu des changements climatiques, qui sous-tend aujourd'hui les logiques de fiscalité écologique, soit récent dans l'histoire industrielle, l'idée de taxer l'énergie ou ses dérivés afin d'éviter les gaspillages et l'épuisement des ressources est ancienne. On la trouve notamment dans la littérature économique du milieu du $\mathrm{XIX}^{\mathrm{e}}$ siècle, au moment où les observateurs de la révolution industrielle prennent conscience que le développement des manufactures implique une utilisation toujours croissante des ressources du sol et du sous-sol. C'est l'âge du charbon en GrandeBretagne et en Europe continentale. Pour ne prendre qu'un exemple, entre 1775 et 1865 , la production charbonnière britannique passe de 8,9 millions de tonnes à 102,3 millions de tonnes [Flinn, 1984 ; Church, 1986] : une multiplication par onze, titanesque !

William Stanley Jevons (1835-1882), professeur d'économie à Manchester puis à Londres au cours des décennies 1860-1880, est l'un des observateurs de l'époque. Quelques années avant d'écrire son traité d'économie politique fondamental, The Theory of Political Economy [1871], qui donnera naissance au marginalisme ${ }^{1}$, il décide en 1865 de consacrer une longue étude monographique à la question du charbon: The Coal Question. An

\footnotetext{
${ }^{1}$ Cofondé par l'Anglais William Stanley Jevons, l'Autrichien Carl Menger et le Français Léon Walras, le marginalisme est un mode de raisonnement économique qui insiste sur le calcul «à la marge », à partir de la notion d'utilité marginale. Ces auteurs indiquent que la valeur d'un bien dépend de l'utilité tirée de la consommation d'une unité supplémentaire du bien, et non de la consommation totale ou moyenne.
}

Inquiry Concerning the Progress of the Nation, and the Probable Exhaustion of Our Coal Mines. Dans son ouvrage, qui connaît une deuxième édition dès 1866, il synthétise l'état des savoirs géologiques sur les réserves houillères encore disponibles, il propose son propre diagnostic sur l'évolution passée et future de la consommation énergétique, il récuse l'optimisme des ingénieurs de son temps qui témoignent d'une confiance selon lui excessive dans l'amélioration future des procédés de production, et il fait part de ses craintes pour l'avenir de l'économie britannique, bientôt asphyxiée par un minerai devenant rare et donc onéreux.

Si les apports de Jevons, dans The Coal Question, sont relativement modestes sur le plan de la théorie économique, ils sont d'un intérêt inattendu pour le lecteur du $\mathrm{XXI}^{\mathrm{e}}$ siècle s'interrogeant sur les enjeux de la fiscalité écologique. Jevons consacre en effet une courte partie de son étude à évaluer les tenants et aboutissants, avantages et inconvénients, de la mise en place d'une taxe générale sur le charbon, évoquée dans les milieux politiques pour diminuer l'usage du minerai afin d'en freiner l'épuisement. Effet de résonance surprenant, Jevons investit en 1865 autant de terrains argumentatifs que ceux que nous rencontrons aujourd'hui. Il évoque les enjeux environnementaux, sociaux, économiques, administratifs et politiques de la mise en place d'une taxe sur le charbon, et ce, dans des directions qui, pour nombre d'entre elles, peuvent nous paraître familières. Son objet, l'épuisement des ressources, est en un sens différent des enjeux climatiques d'aujourd'hui, mais il n'en est pas totalement isolé, tant l'imbrication des problématiques écologiques est importante. Les réflexions de Jevons peuvent nous permettre de prendre un recul historique salvateur dans un contexte où l'immédiateté des préoccupations prime souvent sur les décisions de long terme. Revue de détail.

\section{L'objectif environnemental... et économique de la fiscalité écologique}

Au moment où Jevons écrit, la Grande-Bretagne est la première puissance productrice et exportatrice de charbon. Réputé pour son abondance et sa bonne qualité, le minerai britannique sert alors de multiples usages domestiques et industriels 
(chauffage, force motrice, machinerie) et se trouve exporté aux quatre coins du monde. Pour Jevons, les particularités du charbon britannique en font une richesse naturelle inestimable et irremplaçable. Dès les premières lignes de The Coal Question, il met en évidence cette singularité : "Chaque jour qui passe nous fait voir davan- tage combien le charbon [...] que nous possédons sert de levier majeur à notre civilisation matérielle » [Jevons, 1866, p. 1].

Alors que certains commencent à évoquer la possibilité d'exploiter du pétrole ${ }^{2}$ ou de développer l'électricité, Jevons défend l'idée que le charbon n'admet aucun substitut [Jevons, 1866, p. 165-166], soit pour des raisons de rendements énergétiques inégalables [p. 156-157, p. 163], soit pour des raisons techniques ou logiques : l'électricité permet de transporter de l'énergie, mais cette énergie a dû être au préalable produite à partir de charbon, elle complète mais ne remplace pas le minerai [p. 140141]. Si l'histoire a donné tort à Jevons, avec une substitution progressive $\mathrm{du}$ charbon par les hydrocarbures puis le nucléaire, sa position s'explique par l'état des techniques dans les années 1860 et par la dépendance très forte de tout l'appareil productif britannique envers la pierre noire. Le diagnostic de l'époque est relativement simple : le charbon est le levier du développement économique, il s'épuise, il est donc nécessaire de trouver un moyen de le préserver. L'idée de sauvegarde environnementale occupe une place marginale dans les discours : c'est avant tout pour des motifs économiques que les ressources naturelles sont appelées à être protégées.

Parmi les moyens évoqués pour cette préservation, l'instauration d'une taxe sur le charbon est parfois suggérée, avec une assiette plus ou moins large, de la fiscalisation de toutes les extractions au simple relèvement de certains droits à l'exportation. Jevons voit des avantages incontestables dans la mise en place d'une telle fiscalité : ralentir l'extraction des ressources, et donc en ralentir l'épuisement, fournir de nouvelles recettes aux autorités publiques, ou encore fragiliser les éventuelles industries étrangères qui auraient besoin de minerai britannique [p.355]. Ces arguments - au moins les deux premiers - ne sont pas sans rappeler ceux des défenseurs de la mise en

\footnotetext{
${ }^{2}$ Le premier puits de pétrole à vocation industrielle est mis en activité à Titusville (Pennsylvanie) en 1859 [cf. Copinschi, 2012].
}

place d'une fiscalité carbone au $\mathrm{XXI}^{\mathrm{e}}$ siècle. La préservation des espaces et ressources naturels, ainsi que la constitution d'un fonds d'investissement vert à partir des recettes fiscales obtenues sont en effet des éléments récurrents des discours pro-fiscalité écologique. Dans le cadre qui est le sien, Jevons n'est cependant pas totalement convaincu de l'intérêt à long terme et de la faisabilité immédiate d'une taxe sur le charbon. Avec, là encore, des contre-arguments qui sont loin de nous être inconnus.

\section{Un risque de perte en compétitivité}

Première difficulté selon Jevons, l'instauration d'une taxe peut poser un problème de renchérissement des coûts de production. Dans la mesure où le charbon est à la base de tous les procédés industriels, il représente un coût diffus qui concerne l'ensemble des activités économiques. Renchérir le prix du charbon par une taxe revient à renchérir le prix de tous les biens : "à travers le charbon, nous serions taxés pour tout et à tout instant » [p. 361]. Ce renchérissement est même cumulatif, car on a besoin de charbon à chaque étape du processus de production d'un bien. Cela démultiplie le poids de la fiscalité : "la plupart des choses que nous consommons seraient taxées et retaxées à l'infini à chaque étape de la production » [ibid.]. Résultat: ce ne seraient pas seulement les coûts de production de l'industrie lourde qui seraient renchéris, mais l'ensemble des prix des biens. En termes modernes, Jevons indique ici qu'une taxe sur le seul charbon reviendrait en fait à une perte généralisée de pouvoir d'achat pour l'ensemble des consommateurs. L'économie tout entière en souffrirait.

Doit-on, au nom de la sauvegarde d'une ressource, fragiliser l'ensemble des secteurs économiques? Jevons a des doutes à ce sujet, et ses doutes rejoignent les arguments de ceux qui, aujourd'hui, mettent en exergue l'impact récessif de la mise en place d'une contribution carbone. L'institut Coe-Rexecode indiquait ainsi dans un document de travail d'avril 2013 que la mise en place d'une taxe carbone aurait "un effet nettement récessif : le produit intérieur brut (PIB) [serait] inférieur de $0,03 \%$ à $0,17 \%$ au scénario de référence sans taxe carbone » [Scapecchi, 2013, p. 16]. Ces préoccupations macroéconomiques peuvent paraître comme des effets collatéraux d'un 
instrument fiscal à la prétention plus fondamentale : sauvegarder l'environnement. Mais dans le contexte où écrivait Jevons, où seule la question de la prospérité nationale avait une légitimité, il n'est pas surprenant que ces préoccupations soient au cœur des discussions. Doivent-elles surgir aujourd'hui dans un contexte historique qui a depuis largement évolué, et qui a fait une place certaine aux préoccupations environnementales? La question reste ouverte.

Sur la même thématique, mais à un autre niveau, Jevons laisse entrevoir ses inquiétudes quant à la mise en place d'une taxe sur le charbon à l'échelle du seul territoire britannique. S'il n'en parle pas directement en termes de perte de compétitivité, ses préoccupations sont bien là. Le renchérissement général du prix de tous les biens, à partir de la mise en œuvre d'une taxe sur le charbon, aurait pour conséquence de rendre les produits manufacturés britanniques plus chers [Jevons, 1866, p. 360], et donc plus difficiles à exporter par rapport aux biens français, allemands ou même américains. Sur les marchés mondiaux, les atouts industriels de la Grande-Bretagne se trouveraient fragilisés [p. 74]. En ce sens, la taxe sur le charbon ne permet pas d'éviter les difficultés économiques résultant de l'épuisement des ressources naturelles (hausse des prix énergétiques, perte de pouvoir d'achat), elle ne fait qu'anticiper l'avènement de ces difficultés, en renchérissant le prix du charbon avant même que l'épuisement ne s'en charge.

La thématique de la compétitivité, esquissée ici par Jevons, est aujourd'hui un argument majeur des détracteurs de la contribution carbone. En mars 2010, lorsque le gouvernement français a décidé d'abandonner son projet de taxe carbone, il l'a fait en usant de cet argument, comme en témoignent les propos du Premier ministre François Fillon devant les députés de l'Assemblée nationale : «les décisions que nous prendrons en matière de développement durable doivent être mieux coordonnées avec l'ensemble des pays européens, afin de ne pas creuser [notre] déficit de compétitivité $\gg{ }^{3}$. Loin de Jevons toute idée de coordination internationale en matière de fiscalité écologique. Cependant, sa rhétorique en termes de fragilisation d'une économie nationale par rapport aux concurrents semble avoir survécu depuis presque cent cinquante ans.

\footnotetext{
${ }^{3}$ Réponse de François Fillon à Jean-François Copé, 23 mars 2010 (disponible sur www.assemblee- nationale.fr).
}

Dans la première version du projet français de 2009, une vaste exonération de taxe carbone était prévue pour les industries les plus exposées, par ailleurs soumises au système européen de permis négociables. C'est ce type de mesures dérogatoires qui a été jugé inadéquat par le Conseil constitutionnel. Il connaît pourtant, lui aussi, une histoire ancienne. Jevons indique ainsi que, dès la fin du XVIII ${ }^{\mathrm{e}}$ siècle, des projets de taxe sur le charbon comportaient déjà des exonérations, comme celui présenté par l'homme politique britannique William Pitt en 1784. Ce projet prévoyait une faible taxe sur la consommation de charbon, avec exemption pour «tous les ateliers consommant de grandes quantités de minerai» [Jevons, 1866, p. 362]. Là encore, la fiscalité écologique du $\mathrm{XXI}^{\mathrm{e}}$ siècle ne semble pas se caractériser par un renouveau historique profond.

Sur le terrain économique et concurrentiel, les réflexions de Jevons font donc office d'éclairage instructif. L'énergie est un sujet économiquement stratégique, qu'il s'agisse d'extraction minière au $\mathrm{XIX}^{\mathrm{e}}$ siècle ou de combustion de carbone au $\mathrm{XXI}^{\mathrm{e}}$ siècle. Dans les deux cas, le renchérissement énergétique produit par la taxation engendre des inquiétudes en termes de pouvoir d'achat, mais aussi de coûts de production et de compétitivité. Cette rhétorique fait figure de permanence historique, à laquelle les défenseurs de la fiscalité écologique ne semblent pas, pour l'heure, avoir opposé d'arguments suffisamment solides pour la discréditer.

\section{Des obstacles administratifs}

Exonérer certaines industries d'une taxe écologique peut poser des problèmes politiques, comme la censure par le Conseil constitutionnel de la contribution carbone l'a montré en France ${ }^{4}$. Mais cela peut également poser des problèmes d'ordre pratique, dans les dispositifs administratifs à mettre en place pour déterminer qui peut être exonéré ou non, et pour contrôler que les exonérations ne soient pas abusives. Jevons soulignait déjà ce point en rappelant qu'il est très compliqué pour les autorités publiques de déterminer à chaque instant

\footnotetext{
${ }^{4} \mathrm{C}$ 'est essentiellement pour le non-respect du principe d'égalité devant l'impôt que le Conseil constitutionnel a rejeté le dispositif français de taxe carbone, dans sa décision du 29 décembre 2009 [Conseil constitutionnel, 2009].
} 
si telle ou telle tonne de charbon doit être taxée, en fonction de l'utilisation industrielle à laquelle elle est destinée: "faire la différence entre deux [morceaux de] charbon selon l'usage que l'on en fait serait une tâche difficile voire impossible pour le Trésor public» [Jevons, 1866, p. 362]. D'ailleurs, les obstacles administratifs ne s'arrêtent pas là, selon Jevons. Le charbon existe sous de nombreuses formes et avec des qualités diverses. Cela a-t-il un sens de taxer uniformément toutes ces variétés, au risque de rendre non rentables certains gisements au profit d'autres? Jevons n'en est pas convaincu, par crainte de ce que nous appellerions aujourd'hui des distorsions de marché : «le charbon diffère tant en composition, en qualité et en taille qu'une taxe uniforme serait prohibitive pour l'usage des petits minerais de qualité inférieure, de vastes quantités seraient ainsi perdues et brûlées sur les terrils » [ibid.]. Une taxe ne doit pas privilégier certains acteurs économiques au détriment d'autres, elle doit avoir un poids relatif homogène pour l'ensemble des intervenants d'un même marché. Son objectif est de modifier le comportement de tous les agents, et non pas de donner l'occasion à certains d'améliorer leur position concurrentielle sans effort. Pour éviter ces distorsions, il faudrait taxer de manière différenciée les différents minerais, avec toutes les implications bureaucratiques que cela représenterait, d'où un certain scepticisme de la part de Jevons sur la viabilité d'un tel dispositif [ibid.].

Ce type d'arguments en termes de faisabilité administrative a été récemment mobilisé lorsqu'il s'est agi de réfléchir en Europe sur une taxation des biens selon leur intensité carbone, une intensité difficile à mesurer, en particulier lorsqu'il s'agit de biens importés de l'étranger. La Banque mondiale s'en faisait l'écho, en 2010, en évaluant les obstacles à l'instauration de barrières douanières à dessein écologique : "il serait [...] extrêmement difficile de mesurer exactement le contenu carbone [des biens importés] et les résultats obtenus pourraient être contestés» [Banque mondiale, 2009, p. 252]. Là encore, les points communs entre les discussions du milieu du XIX ${ }^{\mathrm{e}}$ siècle et celles d'aujourd'hui apparaissent au grand jour. Après la rhétorique économique, c'est donc la rhétorique administrative qui fait figure, elle aussi, de permanence historique à l'égard du sujet qui nous intéresse.

\section{Un besoin de redistribution}

Elément plus sensible encore, la question de la justice sociale n'est pas étrangère aux réflexions sur la fiscalité écologique. Dans The Coal Question, Jevons tient à justifier son travail sous deux angles : un angle économique, en avertissant ses contemporains de la menace que représente un épuisement des ressources naturelles pour la prospérité britannique, et un angle social, en mettant en exergue les problèmes de justice intergénérationnelle que peut poser l'exploitation présente des richesses naturelles, au détriment des usages qui pourraient en être faits par les générations à venir. En effet, Jevons rappelle que les ressources naturelles n'appartiennent pas à une génération plutôt qu'à une autre. Or, pour nombre de ces ressources, dont le charbon, si elles sont exploitées aujourd'hui, elles ne pourront plus l'être demain : un gisement épuisé l'est pour de bon. La réflexion sur la préservation des ressources est ici indissociable d'une réflexion en termes de justice entre les générations.

Quelle est la place, dans ce schéma, de la fiscalité écologique? Sur ce point, Jevons décale la perspective par rapport à son évaluation d'une taxe générale sur le charbon. Il en appelle bel et bien aux finances publiques pour assurer une solidarité intergénérationnelle, mais à l'aide d'un instrument très différent, à savoir la réduction de la dette publique : «La seule suggestion que je puisse faire pour compenser [les dommages faits à] la postérité par notre utilisation extravagante du charbon bon marché d'aujourd'hui requiert de l'audace. $J$ 'entends la réduction, voire le remboursement, de la dette publique » [Jevons, 1866, p. 364-365].

Son raisonnement est le suivant. Puisque le charbon s'épuise, il sera de plus en plus cher et représentera un poids toujours plus important pour le développement des générations futures. Les générations actuelles ne peuvent se permettre de laisser en héritage non seulement un charbon onéreux, mais en plus une dette publique importante. Elles se doivent donc de profiter de la prospérité dont elles bénéficient pour, d'une part, assainir les finances publiques, et d'autre part, faire un usage raisonnable des ressources naturelles, comme Jevons le réaffirme en janvier 1867 lors d'une allocution au Carpenters' Hall de Manchester: "Nous devons utiliser nos richesses comme il se doit. Si nous les utilisons en agrément ou à mauvais escient, [...] nous devrons 
simplement être blâmés; mais si nous les utilisons afin d'améliorer la condition de chacun [...] - à travers l'éduca- tion, l'assainissement urbain, [...] l'éradication de la pauvreté, et la construction de bibliothèques et institutions [...] permettant d'assurer la puissance et d'améliorer les aptitudes $d u$ peuple, alors je pense que nous ne serons jamais condamnés pour avoir utilisé notre charbon trop vite » [Jevons, 1867, p. 27-28].

Son analyse quitte ici le terrain étroit de la fiscalité écologique au sens propre, mais elle relie problématique environnementale et redistribution. En appelant la collectivité à mener des politiques d'investissement matériel (infrastructures) et immatériel (éducation), Jevons esquisse ce que nous pourrions nommer un système de redistribution intergénérationnelle : aux générations d'aujourd'hui, l'usage de ressources naturelles abondantes et bon marché ; aux générations futures, l'usage d'infrastructures et d'aptitudes intellectuelles nouvelles. Ce mécanisme se heurte à des problèmes fondamentaux, largement discutés depuis en théorie économique et écologique, notamment sur la possibilité - ou non - de substituer des infrastructures à des ressources naturelles ${ }^{5}$. Mais quoi qu'il en soit, force est d'admettre que Jevons relie sa réflexion environnementale avec des mesures de redistribution, un lien encore aujourd'hui très fort.

Depuis les années 1970-1980 et les premières mises en garde contre les changements climatiques, le discours environnemental s'est structuré autour de la prise en considération du long terme dans les décisions publiques. Pourquoi ? Parce que l'on s'est rendu compte que toute émission de gaz à effet de serre dans l'atmosphère avait un impact non seulement pour aujourd'hui, mais également pour les décennies à venir. Sont alors apparus des objectifs quantifiés de long terme, comme la recommandation du Groupe d'experts intergouvernemental sur l'évolution du climat (Giec) de ne pas provoquer une hausse de la température du Globe supérieure à $2^{\circ} \mathrm{C}$ à horizon 2050. La mise en place de mesures écologiques, notamment fiscales, s'inscrit dans ce type d'objectif qui ne concerne pas seulement les générations présentes, mais également et surtout les générations futures. Les sacrifices opérés aujourd'hui, en termes d'efforts financiers (investissements,

\footnotetext{
${ }^{5}$ C'est le débat de la soutenabilité forte ou faible en économie écologique.
}

taxes énergétiques) et de restrictions techniques (normes écologiques), visent à assurer aux générations de demain un cadre de vie durable. L'idée de solidarité intergénérationnelle occupe donc toujours une place centrale dans les réflexions $\mathrm{du} \mathrm{XXI}^{\mathrm{e}}$ siècle, à l'image des arguments développés au XIX $^{\mathrm{e}}$ siècle. Remarquons d'ailleurs que c'est cette même rhétorique intergénérationnelle qui est mobilisée en Europe depuis les années 2000 pour inviter à la réduction des dettes publiques : chacun appelle ainsi à un effort collectif aujourd'hui pour alléger le «fardeau de la dette » légué aux générations de demain. L'actualité des inspirations de Jevons n'en est que renforcée.

Sur le terrain social, les convergences entre préoccupations du $\mathrm{XIX}^{\mathrm{e}}$ siècle et enjeux du $\mathrm{XXI}^{\mathrm{e}}$ siècle sont tout aussi explicites que sur les terrains économique et administratif. Jevons semble même en avance par rapport aux discours d'aujourd'hui puisqu'il assume le lien entre devenir environnemental et dette publique, deux préoccupations qui restent de nos jours souvent disjointes malgré leurs similitudes en termes de conséquences intergénérationnelles.

\section{De l'épuisement des ressources aux changements climatiques}

Depuis la révolution industrielle, le monde économique a entretenu avec l'environnement des rapports souvent conflictuels. Au XIX siècle, l'épuisement des ressources énergétiques commençait à inquiéter. Dans la première partie du $\mathrm{XX}^{\mathrm{e}}$ siècle, c'est la pollution locale (rivières, brouillard urbain) qui provoquait des troubles à la fois pour les milieux naturels et pour les organismes humains. Dans les années 1960 et 1970, le spectre de l'épuisement des ressources a refait surface, complété dès les années 1980 par les questionnements sur les changements climatiques. Aujourd'hui, c'est de cette panoplie d'inquiétudes que les générations du $\mathrm{XXI}^{\mathrm{e}}$ siècle héritent. Et le temps de la décision politique se fait de plus en plus pressant. La fiscalité écologique est un instrument propice à la confrontation d'idées car elle associe des considérations environnementales, politiques, économiques et sociales. Derrière ces considérations multiples se cache une longue histoire discursive où les arguments d'hier servent parfois encore aujourd'hui alors que les enjeux, eux, ont évolué. 
La position de William Stanley Jevons dans cette histoire est à la fois significative et révélatrice des résurgences argumentatives qui entourent la fiscalité écologique. En 1865, il était question pour lui d'étudier l'épuisement du charbon. Aujourd'hui, les changements climatiques requièrent la mise en place de dispositifs carbone qui restent, pour la plupart, encore à inventer. Pourtant, dans ces deux situations séparées de cent cinquante ans, ce sont les mêmes éléments qui sont mis sur la table pour créditer ou discréditer la mise en place d'une fiscalité. Est-ce légitime, ou du moins cohérent? En un sens oui, car les différents problèmes environnementaux sont liés les uns aux autres: c'est parce que l'on a brûlé un grand volume de minerais et d'hydrocarbures aux $\mathrm{XIX}^{\mathrm{e}}$ et $\mathrm{XX}^{\mathrm{e}}$ siècles que, d'une part, ils s'épuisent, et que, d'autre part, l'atmosphère contient désormais une quantité critique de carbone. Mais derrière cette complémentarité, est-il logique de mobiliser les mêmes arguments pour évaluer une taxe générale sur le charbon en 1865 et une taxe carbone en 2013 ? Voilà qui est discutable.

Une première différence fondamentale vient du fait que le charbon et le carbone n'ont pas le même statut sur le plan marchand. Avant même de parler de taxe, le charbon avait un prix, en tant que ressource achetée et vendue sur un marché. Pour le carbone, les choses sont un peu différentes. Avant de mettre en place des dispositifs publics de taxation ou de marché, le carbone n'avait pas de prix. En conséquence, si la taxe sur le charbon qu'évalue Jevons représente un surcoût par rapport à une valorisation préexistante, une taxe carbone est directement un prix nouveau pour un bien nouveau.

Cette subtilité conceptuelle n'est pas anodine. Pour le charbon, cela signifie que si l'on considère, comme Jevons, qu'à mesure que la ressource s'épuise, son prix augmente, alors une taxe ne fait qu'anticiper l'augmentation future des prix : plutôt que de se produire mécaniquement demain par suite de la raréfaction, l'augmentation des prix se produit artificiellement aujourd'hui par l'instauration de la taxe. D'où les arguments de Jevons sur la précipitation des difficultés économiques qu'une telle augmentation pourrait produire.

Dans le cas du carbone, ce n'est pas la source d'énergie qui est taxée, mais son contenu en carbone. Si l'effet de renchérissement peut paraître identique à celui évoqué précédemment pour des ressources comme les hydrocarbures, il est inexistant pour toutes les énergies non carbonées, exonérées de fait de toute taxe. Par conséquent, une taxe carbone permet non seulement la baisse de la consommation d'énergies carbonées, mais aussi et surtout la promotion des énergies non carbonées. A l'époque de Jevons, le charbon était la seule source d'énergie industriellement viable. Ce n'est pas le cas aujourd'hui. Par conséquent, l'argument selon lequel tous les biens se retrouveraient renchéris par la taxe, au détriment du pouvoir d'achat des consommateurs, est en réalité à nuancer pour le monde que nous connaissons, contrairement à celui de Jevons. Il est possible aujourd'hui de se tourner vers des procédés de production et des biens sans carbone, ou peu carbonés, et d'échapper donc à la taxation. Une différence avec le $\mathrm{XIX}^{\mathrm{e}}$ siècle qui relativise les arguments sur le caractère récessif de la fiscalité écologique contemporaine.

Autre point de divergence entre les enjeux d'hier et d'aujourd'hui, les préoccupations de Jevons étaient, nous l'avons vu, essentiellement - si ce n'est exclusivement - économiques. La prospérité de la Grande-Bretagne était son cheval de bataille, bien avant toute considération sur la préservation des milieux naturels. Aujourd'hui, les changements climatiques représentent un défi d'une ampleur différente, à partir de processus de légitimation moins économiques et plus écologiques. Il en résulte une hiérarchisation des priorités citoyennes a priori nouvelle, où l'économique ne peut être promu au détriment de l'environnemental et du social. Vis-à-vis des réflexions tenues par Jevons, cela signifie que les questions de justice sociale et de redistribution conservent avec pertinence leur actualité dans le domaine de la fiscalité écologique. En revanche, les arguments plus centrés sur la compétitivité et sur la gestion administrative semblent, malgré leur usage courant, prendre trop de poids dans le contexte actuel. Cette méprise ne signifie pas qu'il n'existe aucun argument pertinent mettant en doute la fiscalité écologique, elle dénote simplement que certaines rhétoriques mériteraient d'être mises au second plan en raison de leur inadéquation avec un contexte historique qui a changé.

En fin de compte, l'exemple de William Stanley Jevons comme pionnier des réflexions sur la fiscalité écologique paraît éclairant à plus d'un titre. Eclairant pour déceler la permanence de certains discours qui prêtent aux mesures environnementales des ambitions contraires au développement économique des populations. Eclairant pour souligner les grandes évolutions 
historiques qui ont changé la nature de certaines intentions: si Jevons faisait un lien clair entre fardeau énergétique et fardeau de la dette, ces deux préoccupations sont souvent évoquées séparément aujourd'hui. Eclairant enfin pour mesurer la pertinence de certains arguments à l'égard de défis environnementaux qui ne sont aujourd'hui plus tout à fait les mêmes qu'autrefois : il n'est pas certain que la compétitivité ou le pouvoir d'achat soient exposés avec la même intensité dans le cas d'une fiscalité-charbon que dans le cas d'une fiscalitécarbone. Au-delà, s'il ne fallait retenir qu'un enseignement du travail pionnier de Jevons, ce serait sans doute le suivant: toute décision en matière énergétique et environnementale requiert de l'«audace» [Jevons, 1866, p. 365]; le volontarisme politique fait trop souvent défaut face à des enjeux qui nous dépassent tous individuellement, mais auxquels nous devons collectivement apporter des réponses.

\section{Bibliographie}

Banque mondiale. 2009. Rapport sur le développement dans le monde 2010. Développement et changement climatique. Washington DC, World Bank/Pearson Education.

Church, Roy. 1986. The History of the British Coal Industry, vol. 3: 1830-1913: Victorian Pre- eminence. Oxford : Clarendon Press.

Conseil Constitutionnel. 2009. «Décision n²009599 DC du 29 décembre $2009 »$. Disponible sur www.conseil-constitutionnel.fr.

Copinschi, Philippe. 2012. Le pétrole: une ressource stratégique. Paris: Documentation Française.

Flinn, Michael W. 1984. The History of the British Coal Industry, vol. 2: 1700-1830: The Industrial Revolution. Oxford : Clarendon Press.

Jevons, William S. 1866. The Coal Question; An Inquiry Concerning the Progress of the Nation and the Probable Exhaustion of Our Coal Mines, $2^{\mathrm{e}}$ ed. Londres : Macmillan.

Jevons, William S. 1867/1981. “On Coal.” Lecture at Carpenters' Hall in Manchester, January 16. In Papers and Correspondence of William Stanley Jevons, vol. 7: Papers on Political Economy, edited by R. D. C. Black. Londres : Macmillan.

Jevons, William S. 1871. The Theory of Political Economy. Londres : Macmillan.

Scapecchi, Pascale. 2013. "L'impact sur les secteurs industriels de l'introduction d'une taxe carbone en France» Working paper 39. Paris : Coe-Rexecode. 